\title{
Echocardiographic study of significance of left ventricular minor axis shortening during pre-ejection phase of systole
}

\author{
P. A. N. ChandRaRATNA, G. VLAHOVICH, AND W. S. ARONOW \\ From the Division of Cardiology and Medical Service, Long Beach Veterans Administration Hospital, \\ The University of California, Irvine; and the University of Oklahoma Health Sciences Center, Oklahoma \\ City, Oklahoma, USA
}

SUMMARY This study was designed to investigate the changes in dimensions of the left ventricular minor axis that occur during the pre-ejection period of systole. Three groups of subjects were studied: 29 normal subjects (group 1); 16 patients with mitral regurgitation and 2 patients with ventricular septal defect (group 2); 11 patients with left ventricular volume overload (excluding those with mitral regurgitation and ventricular septal defect), 10 with aortic regurgitation, and 1 witharteriovenous fistula (group 3 ). To define the onset of left ventricular contraction (mitral valve closure), the electrocardiogram and mitral valve echocardiogram were recorded, and to establish the onset of ejection (aortic valve opening), the electrocardiogram and aortic valve were recorded at a paper speed of $50 \mathrm{~mm}$ per second, with $40 \mathrm{~ms}$ time lines. The left ventricular echogram and electrocardiogram were then recorded, and the dimensions of the left ventricular minor axis at the onset of contraction and of ejection were measured. In group 1 the dimension of the left ventricular minor axis either did not change (14 subjects) or increased by 1 to $2 \mathrm{~mm}$ ( 15 subjects) during the pre-ejection period. In group 2, the dimension of the left ventricular minor axis decreased in all the subjects during the pre-ejection period (mean $=3 \mathrm{~mm}$ ). The decrease in the dimension was the result of posterior motion of the interventricular septum during the pre-ejection period; there was no anterior motion of the posterior wall until the onset of ejection except in 1 patient. In group 3 there was no change in the dimension of the left ventricular minor axis during the pre-ejection period except in 1 patient whose dimension increased by $2 \mathrm{~mm}$. We conclude that shortening of the dimension of the left ventricular minor axis during the pre-ejection period occurs in mitral regurgitation and ventricular septal defect, but not in normal subjects or patients with other forms of left ventricular volume overload which are not accompanied by loss of left ventricular volume into another cardiac chamber during the pre-ejection phase of systole. Left ventricular minor axis shortening during the preejection period should be a useful adjunct to the echocardiographic diagnosis of mitral regurgitation and ventricular septal defect.

Conflicting results have been obtained by investigators who have studied the changes that occur in the left ventricular minor axis dimension during the preejection phase of systole (Rushmer, 1954; Karliner et al., 1971; Vokonas et al., 1973). The experimental studies of Rushmer indicated that there was an increase in minor axis diameter during isovolumic contraction (Rushmer, 1954). Vokonas and associates studied the dynamic geometry of the left

Received for publication 6 June 1978 ventricle with left ventricular cineangiography (Vokonas et al., 1973). They observed that there was no significant change in transverse axis dimension during isovolumic contraction. This investigation was designed to study the changes in echocardiographic left ventricular minor axis dimensions during the pre-ejection phase of systole, (i) in normal subjects, (ii) in subjects with mitral regurgitation and ventricular septal defect, and (iii) in patients with other forms of left ventricular volume overload who do not have a loss of left ventricular blood into another cardiac chamber in systole. 


\section{Subjects and methods}

The patients were in the supine or left lateral decubitus position during the echocardiographic examination. An Ekoline 20 ultrasonoscope, a $2 \cdot 25$ $\mathrm{MHz} 10 \mathrm{~cm}$ focus 0.5 in transducer, and Honeywell 1856 or Irex recorder were used. The echocardiograms were recorded at a paper speed of $50 \mathrm{~mm} / \mathrm{s}$, with 40 or $100 \mathrm{~ms}$ time lines.

The left ventricular echocardiogram (with clear delineation of the left septal and left ventricular posterior wall endocardial echo) was recorded using standard techniques. The left ventricle was recorded at the level of the chordae tendineae, except in a 6-month-old baby on whom the recording was made at the level of the mitral leaflets (since this is the standard position for infants). The echocardiogram of the anterior and posterior leaflets of the mitral valve was then recorded, after which echoes from the aortic valve were obtained.

The time interval ( $Q M C$ ) from the $Q$ wave of the electrocardiogram to coaptation of the mitral leaflets

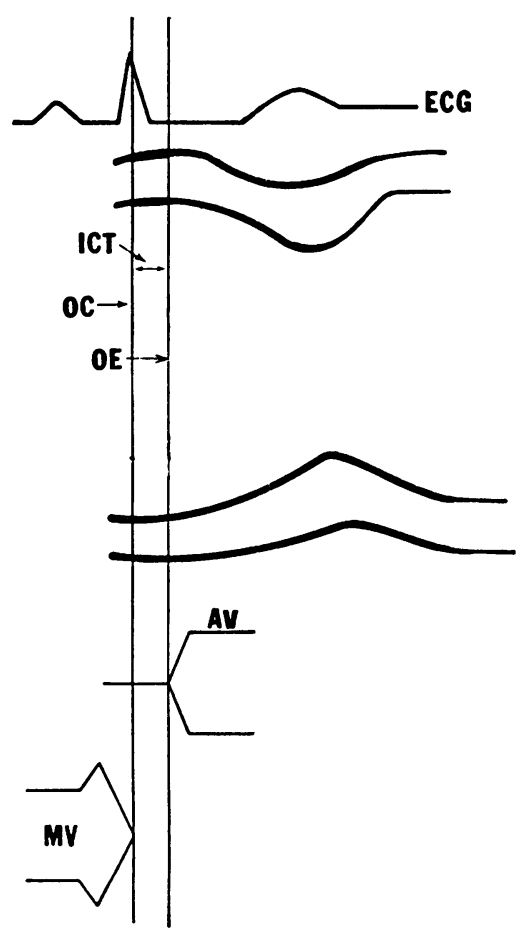

Fig. 1 Diagrammatic representation of the left ventricle, aortic valve $(A V)$, and mitral valve (MV). Mitral closure occurs at the onset of left ventricular contraction $(O C)$, and onset of ejection (OE) occurs at the time of aortic valve opening. ICT $=$ isovolumic contraction time. was measured. Hirschfeld and co-workers (1976) demonstrated that the coaptation of mitral leaflets occurred at the point of rapid increase in left ventricular pressure. Hence the onset of contraction could be defined by measuring the QMC interval. The time interval from the $Q$ wave of the electrocardiogram to aortic valve opening (Q-Ao) was used to determine the onset of ejection. Left ventricular internal dimension was measured at the point of onset of contraction (Fig. 1). Measurements of mitral valve closure, aortic valve opening, and left ventricular dimensions were made at similar $R R$ intervals from 5 separate complexes and average values obtained. It should be noted that beat-tobeat variation of the QMC and Q-Ao interval at similar RR intervals was small (about $5 \mathrm{~ms}$ ). The change in minor axis dimension during the preejection phase of left ventricular contraction equals the dimension at the onset of contraction minus the dimension at the onset of ejection. (The pre-ejection phase of contraction is the same as isovolumic systole for normal subjects but in patients with mitral regurgitation there may be no isovolumic systole; therefore the former term was used.)

In 2 patients (one with severe mitral regurgitation and another with severe aortic regurgitation) simultaneous left ventricular echograms and central aortic pressure (using a micromanometer-tipped catheter) were recorded at paper speeds of 50 and $100 \mathrm{~mm} / \mathrm{s}$ and $40 \mathrm{~ms}$ time lines.

Three groups of patients were studied. Group 1 comprised 29 normal subjects whose ages ranged from 20 to 40 years. There were 12 women. Group 2 comprised 18 patients with left ventricular volume overload who had loss of left ventricular volume into another cardiac chamber (right ventricle or left atrium) during the pre-ejection phase of systole; 16 had mitral regurgitation documented by left ventricular cineangiography and 2 had uncomplicated ventricular septal defects with left-toright shunts (QP:QS of 2.8 and 3, respectively). All the patients with mitral regurgitation had moderate (grade 3/4) or severe (grade 4/4) regurgitation, except case 14 who had grade $1 / 4$ regurgitation and case 13 who had grade 2/4 reflux. Group 3 consisted of 10 patients with moderate or severe aortic regurgitation as assessed by aortic root angiography and 1 patient with a fistula between the left iliac artery and vein (after laminectomy). These patients had types of left ventricular overload which were not associated with loss of left ventricular blood into another cardiac chamber during the pre-ejection period.

\section{Results}

The results are outlined in Tables 1,2 , and 3 . The 
data from normal subjects are shown in Table 1. The left ventricular minor axis dimensions at the onset of contraction (mitral valve closure) ranged from 36 to

Table 1 Echocardiographic data in normal subjects

\begin{tabular}{|c|c|c|c|c|c|}
\hline Case no. & $\begin{array}{l}\text { Age } \\
\text { (y) }\end{array}$ & Sex & $D O C$ & $D O E$ & $\triangle D-P E P$ \\
\hline 1 & 25 & $\mathbf{F}$ & 42 & 43 & -1 \\
\hline 2 & 22 & $\mathbf{F}$ & 38 & 38 & 0 \\
\hline 3 & 23 & F & 45 & 45 & 0 \\
\hline 4 & 24 & $\mathbf{M}$ & 54 & 54 & 0 \\
\hline 5 & 26 & $\mathbf{M}$ & 46 & 47 & -1 \\
\hline 6 & 29 & $\mathbf{M}$ & 54 & 54 & 0 \\
\hline 7 & 25 & $\mathbf{F}$ & 37 & 38 & -1 \\
\hline 8 & 25 & $\mathbf{M}$ & 46 & 48 & -2 \\
\hline 9 & 24 & $\mathbf{M}$ & 41 & 41 & 0 \\
\hline 10 & 26 & $\mathbf{M}$ & 48 & 50 & -2 \\
\hline 11 & 21 & $\mathbf{F}$ & 40 & 40 & 0 \\
\hline 12 & 22 & $M$ & 49 & 49 & 0 \\
\hline 13 & 23 & $\mathbf{M}$ & 44 & 44 & 0 \\
\hline 14 & 20 & $\mathbf{F}$ & 48 & 48 & 0 \\
\hline 15 & 27 & $\mathbf{F}$ & 42 & 42 & 0 \\
\hline 16 & 22 & $M$ & 49 & 49 & 0 \\
\hline 17 & 38 & $\mathbf{F}$ & 46 & 47 & -1 \\
\hline 18 & 32 & $\mathbf{M}$ & 48 & 48 & 0 \\
\hline 19 & 22 & $\mathbf{F}$ & 39 & 40 & -1 \\
\hline 20 & 40 & F & 52 & 52 & 0 \\
\hline 21 & 25 & $\mathbf{F}$ & 46 & 47 & -1 \\
\hline 22 & 20 & $\mathbf{F}$ & 36 & 37 & -1 \\
\hline 23 & 31 & $\mathbf{M}$ & 52 & 54 & -2 \\
\hline 24 & 29 & $\mathbf{M}$ & 47 & 49 & -2 \\
\hline 25 & 33 & $\mathbf{M}$ & 50 & 51 & -1 \\
\hline 26 & 23 & $\mathbf{M}$ & 48 & 48 & 0 \\
\hline 27 & 21 & $\mathbf{M}$ & 51 & 52 & -1 \\
\hline 28 & 31 & $\mathbf{M}$ & 45 & 47 & -2 \\
\hline 29 & 25 & $\mathbf{M}$ & 39 & 40 & -1 \\
\hline
\end{tabular}

DOC, left ventricular minor axis dimension in $\mathrm{mm}$ at the onset of contraction; DOE, left ventricular minor axis dimension in $\mathrm{mm}$ at the onset of ejection; $\triangle \mathrm{D}-\mathrm{PEP}=\mathrm{DOC}-\mathrm{DOE}$, that is change in minor axis dimension during the pre-ejection phase of systole. A negative value signifies an increase in minor axis diameter during the pre-ejection period.
Table 3 Aortic regurgitation

\begin{tabular}{llllll}
\hline Case no. & Age & Sex & DOC & DOE & $\triangle D-P E P$ \\
\hline 1 & 32 & M & 72 & 72 & 0 \\
2 & 41 & F & 58 & 58 & 0 \\
3 & 42 & M & 57 & 57 & 0 \\
4 & 44 & M & 68 & 68 & 0 \\
5 & 52 & M & 60 & 60 & 0 \\
6 & 31 & M & 75 & 75 & 0 \\
7 & 27 & M & 59 & 61 & -2 \\
8 & 17 & M & 67 & 67 & 0 \\
9 & 76 & F & 60 & 60 & 0 \\
10 & 52 & M & 63 & 63 & 0 \\
Arteriovenous fistula & M & 57 & 57 & 0 \\
1 & 36 & M & &
\end{tabular}

$54 \mathrm{~mm}$. The minor axis dimension at the onset of ejection ranged from 37 to $54 \mathrm{~mm}$. None of the normal subjects showed shortening of the minor axis dimension during the pre-ejection period. Fourteen patients did not exhibit a change in minor axis dimension during the pre-ejection phase of systole. The diameter of the minor axis increased by 1 to $2 \mathrm{~mm}$ in 15 subjects. Data from patients with mitral regurgitation and ventricular septal defect are given in Table 2. All 16 patients with mitral regurgitation showed minor axis shortening during the pre-ejection period. Shortening of the minor axis diameter during the pre-ejection period was the result of posterior motion of the interventricular septum (range 2 to $4 \mathrm{~mm}$ ); there was no anterior motion of the left ventricular posterior wall except in 1 patient. The percentage minor axis shortening during the pre-ejection period ranged from $3 \cdot 3$ to $7 \cdot 2$ per cent, with a mean of 5.5 per cent.

Data obtained from patients with aortic regurgita-

Table 2 Echocardiographic data in mitral regurgitation and in ventricular septal defect

\begin{tabular}{|c|c|c|c|c|c|c|c|c|}
\hline Case no. & Age & Sex & $D O C$ & $D O E$ & $\triangle D-P E P$ & $D-I V S-P E P$ & $D P W-P E P$ & $\% M A S-P E P$ \\
\hline \multicolumn{9}{|c|}{ Mitral regurgitation } \\
\hline 1 & 40 & $\mathbf{M}$ & 62 & 58 & 4 & 4 & 0 & $6 \cdot 4$ \\
\hline 2 & 22 & $\mathbf{F}$ & 68 & 64 & 4 & 4 & 0 & $5 \cdot 8$ \\
\hline 3 & 26 & $\mathbf{M}$ & 67 & 63 & 4 & 4 & 0 & $5 \cdot 9$ \\
\hline 4 & 17 & $\mathbf{M}$ & 70 & 67 & 3 & 3 & 0 & $4 \cdot 2$ \\
\hline 5 & 52 & $\mathbf{F}$ & 55 & 51 & 4 & 4 & 0 & $7 \cdot 2$ \\
\hline 6 & 37 & $\mathbf{F}$ & 58 & 54 & 4 & 4 & 0 & $6 \cdot 8$ \\
\hline 7 & 40 & $\mathrm{~F}$ & 61 & 57 & 4 & 4 & 0 & $6 \cdot 5$ \\
\hline 8 & 56 & $\mathbf{M}$ & 65 & 61 & 4 & 4 & 0 & $6 \cdot 1$ \\
\hline 9 & 18 & $\mathbf{M}$ & 74 & 71 & 3 & 3 & 0 & $4 \cdot 0$ \\
\hline 10 & 47 & $\mathbf{F}$ & 60 & 57 & 3 & 3 & 0 & $5 \cdot 0$ \\
\hline 11 & 53 & $\mathbf{F}$ & 54 & 51 & 3 & 3 & 0 & $5 \cdot 5$ \\
\hline 12 & 31 & $\mathrm{~F}$ & 55 & 52 & 3 & 3 & 0 & $5 \cdot 4$ \\
\hline 13 & 52 & $\mathbf{M}$ & 53 & 50 & 3 & 2 & 1 & $5 \cdot 6$ \\
\hline 14 & 30 & $\mathbf{F}$ & 42 & 40 & 2 & 2 & 0 & $4 \cdot 7$ \\
\hline 15 & 38 & $\mathrm{~F}$ & 60 & 58 & 2 & 2 & 0 & $3 \cdot 3$ \\
\hline 16 & 54 & $\mathbf{M}$ & 58 & 55 & 3 & 3 & 0 & $5 \cdot 2$ \\
\hline \multicolumn{9}{|c|}{ Ventricular septal defect } \\
\hline 1 & $1 \frac{1}{2}$ & $\mathbf{F}$ & 23 & 21 & 2 & 2 & 0 & \\
\hline 2 & $6 \mathrm{mth}$ & $\mathbf{M}$ & 28 & 25 & 3 & 3 & 0 & \\
\hline
\end{tabular}

D-IVS-PEP, posterior displacement (in $\mathrm{mm}$ ) of the interventricular septum during the pre-ejection phase of systole; DPW-PEP, anterior displacement of the left ventricular posterior wall endocardial echo during the pre-ejection phase of the systole. \%MAS-PEP, percentage minor axis shortening during the pre-ejection phase of systole $=\frac{\triangle D-P E P}{D O C} \times 100$. 
tion or arteriovenous fistula are shown in Table 3. All except 1 patient showed no change in minor axis diameter during the pre-ejection phase of systole.

The left ventricular echocardiogram of a normal subject is illustrated in Fig. 2. The minor axis dimension at the onset of ejection is $1 \mathrm{~mm}$ greater than that at the onset of left ventricular contraction. The ultrasound recording of a patient with mitral regurgitation is depicted in Fig. 3. The minor axis diameter at the onset of contraction is $6.0 \mathrm{~cm}$ and that at the onset of ejection is $5.7 \mathrm{~cm}$, indicating that there has been minor axis shortening of $3 \mathrm{~mm}$ during the pre-ejection phase of systole. An echocardiogram of the left ventricle and a simultaneous recording of the central aortic pressure (catheter tip micromanometer) in a patient with mitral regurgitation are presented in Fig. 4. There is posterior motion of the septum resulting in minor axis shortening before the upstroke of the aortic pressure

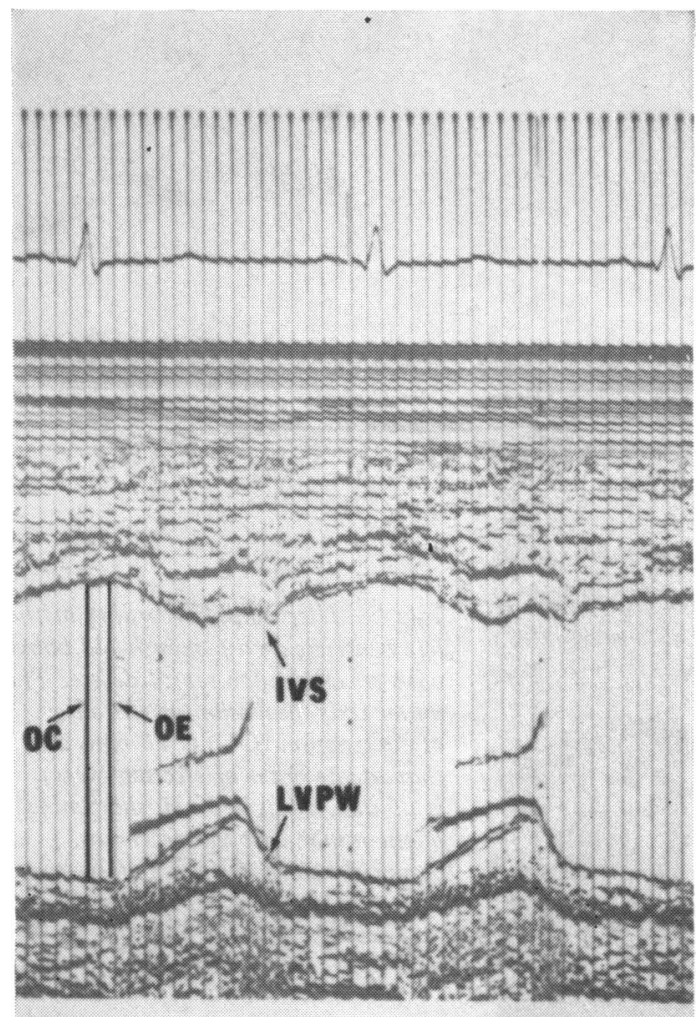

Fig. 2 Echocardiogram of the left ventricle in a normal subject. The minor axis dimension at the onset of contraction $(O C)$ is $46 \mathrm{~mm}$, and that at the onset of ejection $(O E)$ is $47 \mathrm{~mm}$. The minor axis diameter has increased by $1 \mathrm{~mm}$ during the pre-ejection phase of systole.

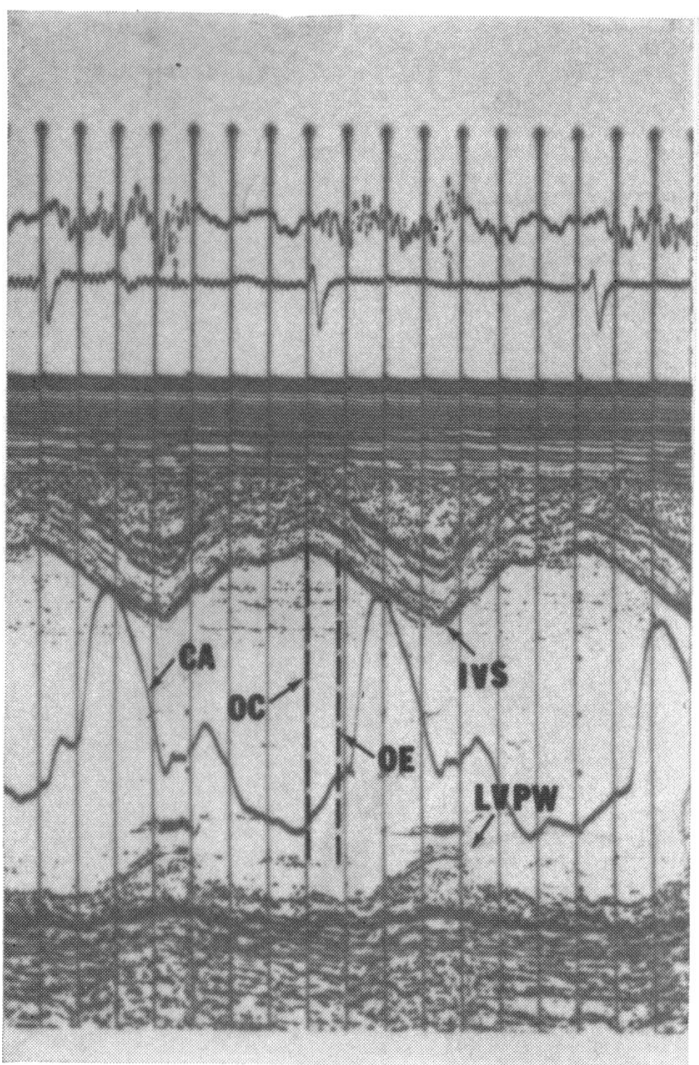

Fig. 3 Echocardiogram of left ventricle from a patient with mitral regurgitation. The left ventricular minor axis diameter is $60 \mathrm{~mm}$ at the onset of contraction, and $57 \mathrm{~mm}$ at the onset of ejection. The minor axis has shortened by $3 \mathrm{~mm}$ during the pre-ejection phase of systole. $A$ carotid pulse (CA) recording was also made in this patient. Note that the onset of the carotid upstroke occurs later than the onset of ejection (as determined by point of aortic valve opening) because of the delay in transmission of the carotid pulse. IVS, interventricular septum; LVPW, left ventricular posterior wall. The time lines are $100 \mathrm{~ms}$ apart. The vertical dotted line OC indicates the timing of the onset of ventricular contraction (not the minor axis dimension, since the line does not extend from the left septal endocardium to the endocardium of the posterior wall), and the line $O E$ indicates the onset of left ventricular ejection.

pulse. A left ventricular echocardiogram from a 6-month-old baby with a ventricular septal defect is illustrated in Fig. 5. Minor axis shortening in the pre-ejection phase of systole is evident.

\section{Discussion}

Left ventricular dilatation with increased excursion of the septum, left atrial dilatation, increased pulsa- 


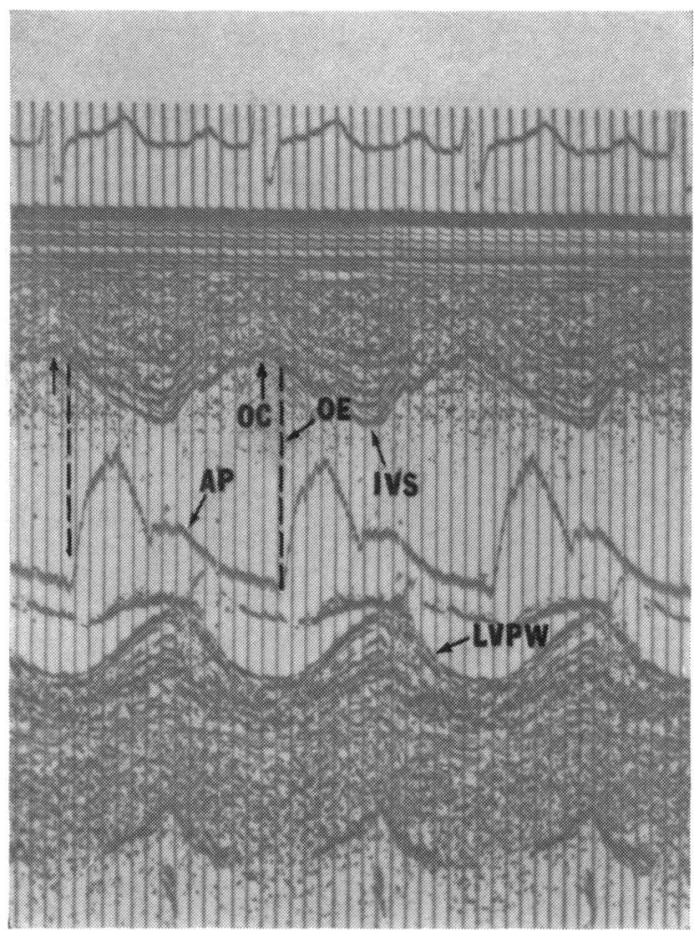

Fig. 4 Simultaneous recording of the left ventricular echocardiogram and aortic pressure $(A P)$ recorded by means of a catheter-tipped micromanometer placed just above the aortic valve in a patient with mitral regurgitation. Note that posterior motion of the septum has occurred before the onset of the upstroke of the aortic pressure pulse (onset of ejection), indicating minor axis shortening in the pre-ejection phase of systole. $O C$, onset of contraction; $O E$, onset of ejection. Time lines are $40 \mathrm{~ms}$ apart.

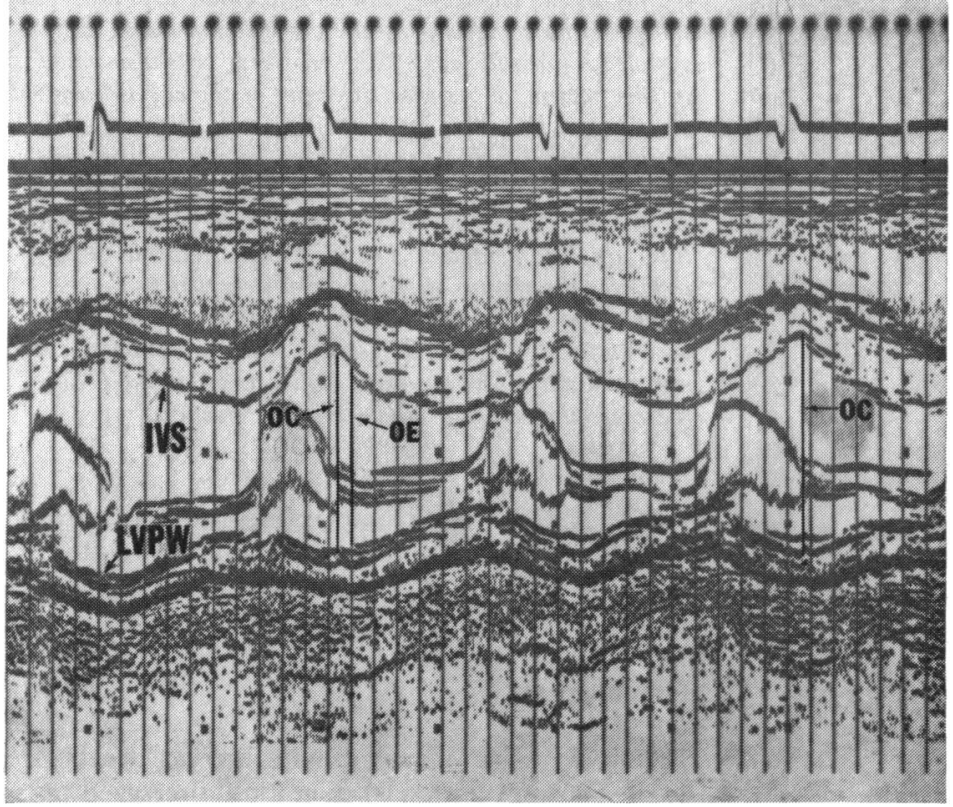

Fig. 5 Ultrasound recording of the left ventricle in a 6-month-old baby with a ventricular septal defect. The minor axis dimension at the onset of contraction (OC) is $28 \mathrm{~mm}$, and that at the onset of ejection (OE) is $25 \mathrm{~mm}$. Thus a $3 \mathrm{~mm}$ shortening of the minor axis diameter has occurred before the onset of ejection. The time lines are $40 \mathrm{~ms}$ apart. 
tion of the left atrial posterior wall, and increased E-F slope of the mitral valve have been noted in the echocardiograms of patients with mitral regurgitation (Feigenbaum, 1976). Many of these ultrasound features may also be seen in other conditions such as aortic regurgitation, arteriovenous fistula, and persistent ductus arteriosus which produce left ventricular volume overload. We, therefore, explored the value of echocardiographically detectable minor axis shortening during the preejection period as a means of differentiating between mitral regurgitation or ventricular septal defect, which are conditions associated with loss of left ventricular blood into another cardiac chamber during the pre-ejection period, and other forms of left ventricular volume overload that do not exhibit left ventricular volume loss in the pre-ejection phase of systole.

Several investigators have studied the dimensional changes in the left ventricle during isovolumic contraction. Though an increase in minor axis diameter during isovolumic contraction has been reported in dog experiments, recent studies in animals and man have failed to confirm this finding (Rushmer, 1954; Karliner et al., 1971; Vokonas et al., 1973). Rankin and associates (1976) indicated that this discrepancy arose because the dynamic geometry of the left ventricle in the open chested dog preparation was different from that in conscious subjects. They pointed out that ventricular volume diminished during thoracotomy, and the tendency of the ventricle to assume a more spherical shape during isovolumic contraction was increased. Karliner and colleagues (1971) noted a mean decrease in transverse diameter of $1 \mathrm{~mm}$ before aortic valve opening in 6 subjects without left ventricular dysfunction. Three of their subjects had organic heart disease, 2 had functional cardiac murmurs, and 1 had atypical chest pain. Vokonas and associates (1973) found no significant changes in left ventricular minor axis dimension of normal subjects, while in patients with mitral regurgitation and good left ventricular function the transverse axis shortened an average of 5.2 per cent during the preejection phase of left ventricular contraction. We observed that the left ventricular minor axis in normal subjects either did not change or increased slightly ( 1 to $2 \mathrm{~mm}$ ) during the pre-ejection period. In contrast, minor axis shortening during the preejection period was noted in all our patients with mitral regurgitation. Venco and associates (1977) noted a reduction in left ventricular minor axis dimensions during the upstroke of the apex cardiogram in patients with mitral regurgitation, reflecting backward movement of blood into the left atrium before aortic valve opening. It is of interest that the mean value of $5 \cdot 2$ per cent for transverse axis shortening during the pre-ejection period in a group of patients with compensated mitral regurgitation reported by Vokonas and co-workers (1973) is similar to that observed $(5.5 \%)$ in our study. Minor axis shortening during the pre-ejection period was also observed in 2 patients with ventricular septal defect who had left-to-right shunts. It is possible that patients who have only right-to-left shunting (Eisenmenger ventricular septal defect) may not show minor axis shortening in the pre-ejection period. Patients with other forms of left ventricular volume overload, who did not have a 'run-off' of left ventricular blood into another cardiac chamber in systole, did not exhibit pre-ejection minor axis shortening.

The diagnosis of mitral regurgitation is critical in certain situations. Patients with severe acute aortic regurgitation secondary to infective endocarditis show premature mitral valve closure, which to a certain extent prevents transmission of the high left ventricular diastolic pressure to the left atrium (Morganroth et al., 1977). The ocurrence of mitral regurgitation in such a patient will, therefore, result in severe haemodynamic deterioration (Morganroth et al., 1977). The early detection of mitral regurgitation in that situation is of paramont importance. Serial echocardiograms to detect minor axis shortening before aortic valve opening will be of value in this setting.

Certain factors may limit the usefulness of preejection minor axis shortening as an index of mitral regurgitation or ventricular septal defect. This echographic sign may not be present in patients who have late systolic mitral regurgitation. It is conceivable that some patients who have abnormal septal motion caused by left bundle-branch block, anteroseptal myocardial infarction, or idopathic hypertrophic subaortic stenosis may not exhibit posterior septal motion in the pre-ejection period, in spite of the presence of significant mitral regurgitation. Vokonas and co-workers (1973) observed that changes in geometry before ejection were less evident in patients who had mitral regurgitation and poor left ventricular performance. Since all our patient had good left ventricular function, the usefulness or limitation of minor axis shortening in the pre-ejection period as a sign of mitral regurgitation in patients with decompensated left ventricles remains to be established. Two patients in our group had mild mitral regurgitation. The role of echocardiography in detecting minor axis shortening during the pre-ejection period in patients with mild mitral regurgitation needs further investigation.

In summary, left ventricular minor axis dimension either does not change or increases slightly during 
the pre-ejection phase of systole in normal subjects and in patients with forms of left ventricular volume overload which are not accompanied by loss of left ventricular volume into another cardiac chamber during the pre-ejection period. In contrast, patients with mitral regurgitation and ventricular septal defect have minor axis shortening in the preejection phase of systole. Echocardiographic left ventricular minor axis shortening in the pre-ejection period should prove to be a valuable adjunct in the diagnosis of mitral regurgitation and ventricular septal defect.

\section{References}

Feigenbaum, H. (1976). Echocardiography. Lea and Febiger, Philadelphia.

Hirschfeld, S., Meyer, R., Korhagen, J., Kaplan, S., and Liebman, J. (1976). The isovolumic contraction time of the left ventricle: an echographic study. Circulation, 54, 751-756.

Karliner, J. S., Bouchard, R. J., and Gault, J. H. (1971).
Dimensional changes of the human left ventricle prior to aortic valve opening. Circulation, 44, 312-322.

Morganroth, J., Perloff, J. K., Zeldis, S. M., and Dunkman W. B. (1977). Acute severe aortic regurgitation. Annals of Internal Medicine, 87, 223-232.

Rankin, J. S., McHale, P. A., Arentzen, C. E., Ling, D., Greenfield, J. C., Jr., and Anderson, R. W. (1976). The three-dimensional dynamic geometry of the left ventricle in the conscious dog. Circulation Research, 38, 304-313.

Rushmer, R. F. (1954). Continuous measurements of left ventricular dimension in intact, unanesthetized dogs. Circulation Research, 2, 14-21.

Venco, A., Gibson, D. G., and Brown, D. J. (1977). Relation between apex cardiogram and changes in left ventricular pressure and dimension. British Heart fournal, 39, 117-125.

Vokonas, P. S., Gorlin, R., Cohn, P. F., Herman, M. V., and Sonnenblick, E. H. (1973). Dynamic geometry of the left ventricle in mitral regurgitation. Circulation, 48, 786-796.

Requests for reprints to Dr P. A. N. Chandraratna, Department of Cardiology, Veterans Administration Hospital, Long Beach, California 90822, USA.

\section{Announcement}

\section{Basic cardiology seminar}

A seminar on basic cardiology will be organised every year by the French Society of Cardiology and sponsored by the European Society.

The first session will be held on 25, 26, and 27 October 1979, at Merck, Sharp \& DohmeChibret Laboratories Centre, 63000 ClermontFerrand (France). It will include two topics: haemodynamic exploration in cardiology, and heart and calcium.

The objective is to establish a dialogue between experts and young cardiologists who want to improve their knowledge of experimental and/or clinical, basic, and/or applied research. The seminar will be bilingual, French-English. Registration and hotel accommodation will be free of charge.

Applicants must: (a) be less than 35 years old; (b) have a perfect knowledge of French or English;

(c) submit a file including: professional training, hospital and university titles, a list of publications, and a reference from the department head.

Applications will be received until 15 May 1979. The number of participants is limited to 90 .

Professeur René Gourgon

Secrétaire du séminaire de cardiologie fondamentale

Hôpital Beaujon,

100 boulevard du Général Leclerc, 92118 Clichy Cedex (France). 